\title{
Constraints on compact binary merger evolution from spin-orbit misalignment in gravitational-wave observations
}

\author{
B. P. Gompertz, ${ }^{1 \star}$ M. Nicholl, ${ }^{1}$ P. Schmidt,${ }^{1}$ G. Pratten, ${ }^{1}$ and A. Vecchio ${ }^{1}$ \\ ${ }^{1}$ School of Physics and Astronomy \& Institute for Gravitational Wave Astronomy, University of Birmingham, Birmingham, B15 2TT, UK
}

Accepted XXX. Received YYY; in original form ZZZ

\begin{abstract}
The identification of the first confirmed neutron star - black hole (NS-BH) binary mergers by the LIGO, Virgo and KAGRA collaboration provides the opportunity to investigate the properties of the early sample of confirmed and candidate events. Here, we focus primarily on the tilt angle of the black hole's spin relative to the orbital angular momentum vector of the binary, and the implications for the physical processes that determine this tilt. The posterior tilt distributions of GW200115 and the candidate events GW190426_152155 and GW190917_114630 peak at significantly anti-aligned orientations (though display wide distributions). Producing these tilts through isolated binary evolution would require stronger natal kicks than are typically considered (and preferentially-polar kicks would be ruled out), and/or an additional source of tilt such as stable mass transfer. The early sample of NS-BH events are less massive than expected for classical formation channels, and may provide evidence for efficient mass transfer that results in the merger of more massive NS-BH binaries before their evolution to the compact phase is complete. We predict that future gravitational-wave detections of NS-BH events will continue to display total binary masses of $\approx 7 \mathrm{M}_{\odot}$ and mass ratios of $q \sim 3$ if this interpretation is correct. Conversely, the high mass of the candidate GW191219_163120 suggests a dynamical capture origin. Large tilts in a significant fraction of merging NS-BH systems would weaken the prospects for electromagnetic detection. However, EM observations, including non-detections, can significantly tighten the constraints on spin and mass ratio.
\end{abstract}

Key words: gravitational waves - stars: black holes - stars: neutron - binaries: general - transients: black hole - neutron star mergers - transients: gamma-ray bursts

\section{INTRODUCTION}

The era of gravitational-wave (GW) astronomy has driven a revolution in our understanding of the physics of compact objects. Since the first discovery of a merging binary black hole (BBH) system (Abbott et al. 2016), the Advanced LIGO (LIGO Scientific Collaboration et al. 2015) and Virgo (Acernese et al. 2015) interferometers have provided a remarkable wealth of information on the denizens of the 'stellar graveyard'. Particularly notable milestones include the identification of the first intermediate mass black hole $(\mathrm{BH}$; Abbott et al. 2020a) and the detection of the landmark GW170817, the first signal from a merging binary neutron star (NS) system (Abbott et al. 2017a). The latter event also yielded the first ever electromagnetic (EM) counterpart to a GW event; the gamma-ray burst (GRB) 170817A (Abbott et al. 2017c; Goldstein et al. 2017; Hallinan et al. 2017; Margutti et al. 2017; Savchenko et al. 2017; Troja et al. 2017; D'Avanzo et al. 2018; Lyman et al. 2018; Margutti et al. 2018; Mooley et al. 2018; Troja et al. 2018; Lamb et al. 2019), and the kilonova (KN) AT2017gfo (Andreoni et al. 2017; Arcavi et al. 2017; Chornock et al. 2017; Coulter et al. 2017; Cowperthwaite et al. 2017; Drout et al. 2017; Evans et al. 2017; Kasliwal et al. 2017; Lipunov et al. 2017; McCully et al. 2017; Nicholl et al. 2017; Pian et al. 2017;

^ E-mail: b.gompertz@bham.ac.uk (BPG)
Shappee et al. 2017; Smartt et al. 2017; Soares-Santos et al. 2017; Tanvir et al. 2017; Utsumi et al. 2017; Valenti et al. 2017; Villar et al. 2017), heralding the advent of GW-EM multi-messenger astronomy (Abbott et al. 2017b).

More recently, the LIGO-Virgo-KAGRA (Kagra Collaboration et al. 2019) collaboration (LVK) announced the detection of the merger of two NS-BH binary mergers (Abbott et al. 2021e), completing the set of compact binary merger constituents. The two binaries were measured to have component masses of $8.9_{-1.5}^{+1.2} \mathrm{M}_{\odot}$ and $1.9_{-0.3}^{+0.3} \mathrm{M}_{\odot}(\mathrm{GW} 200105)$, and $5.7_{-2.1}^{+1.8} \mathrm{M}_{\odot}$ and $1.5_{-0.3}^{+0.7} \mathrm{M}_{\odot}$ (GW200115) assuming agnostic priors for the spin of the secondary. Interestingly, the spin projection of the primary of GW200115 onto the orbital angular momentum was negative with 88 per cent probability, potentially indicating a significant spin-orbit tilt angle. The primary spin magnitude of GW200105 was less than 0.23 , but its direction was unconstrained. In addition to the newly-confirmed events, known NS-BH candidates also include GW190426_152155 and GW190814 (Abbott et al. 2021b), GW190917_114630 (The LIGO Scientific Collaboration et al. 2021a), and GW191219 163120 (The LIGO Scientific Collaboration et al. 2021b). The $2.59_{-0.08}^{+0.09} \mathrm{M}_{\odot}$ secondary of GW190814 (Abbott et al. 2020b) is much more massive than even the heaviest known NSs (Demorest et al. 2010; Antoniadis et al. 2013; Cromartie et al. 2020) and is omitted here as a likely $\mathrm{BBH}$ event. 
The NS-BH variant of compact object mergers garners particular attention because it enables observers to explore the maximum stable mass of an NS, and the lower 'mass gap' between $3-5 \mathrm{M}_{\odot}$ (e.g. Özel et al. 2010; Farr et al. 2011). If the inspiraling NS is disrupted before plunging into the $\mathrm{BH}, \mathrm{EM}$ observations may also be possible (e.g. Kawaguchi et al. 2016; Foucart et al. 2018; Fernández et al. 2020; Krüger \& Foucart 2020). Combined with GW measurements of the mass, EM observations of compact binary mergers can provide multimessenger constraints on the NS equation-of-state (EoS) (Margalit \& Metzger 2017, 2019; Coughlin et al. 2019; Dietrich et al. 2020; Nicholl et al. 2021; Breschi et al. 2021).

The ability to study non-photonic events, previously inaccessible to traditional EM astronomy, has also led to tighter constraints on properties such as merger rates, mass distributions, and binary configurations (Abbott et al. 2021d). In turn, these observations can be used to elucidate the properties of the population of compact binaries prior to the merger. In particular, the mechanism by which the binary was formed may imprint itself on the tilt angle $\theta_{\mathrm{BH}}$; the inclination between the $\mathrm{BH}$ spin and the binary orbital plane. This possibility has been previously explored in e.g. Farr et al. (2017); Stevenson et al. (2017); Talbot \& Thrane (2017); Vitale et al. (2017a); Roulet et al. (2021).

In cases where the binary formed via dynamical capture, whether in dense stellar clusters (e.g. Hoang et al. 2020; Rastello et al. 2020; Ye et al. 2020) or AGN discs (e.g. McKernan et al. 2020b,a), we can expect an isotropic distribution of tilt angles because the spins of the two constituents are independent of one another and the orbital plane is set by the direction of approach during the capture (e.g. Rodriguez et al. 2016). Where the system was formed via isolated binary evolution (e.g. Smarr \& Blandford 1976; Srinivasan 1989; Belczynski et al. 2002), the misalignment is expected to be small because viscous forces will align both the spins and the orbit along the dominant angular momentum plane of the original gas cloud.

However, the identification of significant tilt angles is not necessarily a smoking gun for dynamical capture because several mechanisms still exist by which the spin of one or both of the constituents of a binary formed in isolation may become misaligned with the orbital plane. Possible mechanisms include natal kicks that imbue a significant velocity on the stellar remnant during core collapse (e.g. Rodriguez et al. 2016), or stable mass transfer that may tilt the donor star's spin vector into the orbital plane (e.g. Stegmann \& Antonini 2021). In addition to this, the efficiency with which common envelope $(\mathrm{CE})$ processes and tidal forces can realign the spin and orbit vectors is uncertain.

Broekgaarden \& Berger (2021) demonstrated that the chirp masses, component masses and mass ratios of GW200105 and GW200115 are consistent with an isolated binary evolution channel in their population synthesis modelling. Combined with the low expected rates of dynamical capture-driven NS-BH mergers (e.g. Arca Sedda 2020; Hoang et al. 2020; Ye et al. 2020), we consider the isolated binary formation channel as our preferred pathway. In this paper, we investigate the physical processes that may produce significant spin-orbit anti-alignment, and compare the tilt angles of confirmed or candidate NS-BH mergers to the population of $\mathrm{BBH}$ mergers presented in (Abbott et al. 2021d). In Section 2 we describe the data sample and assess the GW posteriors for the BH tilt angle. We then draw constraints on compact binary evolution in Section 3 and their implications for future observations of compact binaries in Section 4. Finally, we discuss our conclusions in Section 5.

\section{GRAVITATIONAL-WAVE OBSERVATIONS}

To determine whether the $\mathrm{BH}$ spin tilt angles $\theta_{\mathrm{BH}}$ observed in the NS-BH mergers are consistent with the observed astrophysical $\mathrm{BH}$ population, we compare the $\mathrm{BH}$ tilt angle measurements of the NS$\mathrm{BH}$ events to the full GW-detected $\mathrm{BH}$ sample, which consists exclusively of BBH mergers. We construct posterior predictive distributions (PPD) for the spin tilt using the public posteriors from the default spin model (Abbott et al. 2021d,a; Abbott et al. 2021c), treating the spin of each component $\mathrm{BH}$ as being independently drawn from the same underlying distribution. The default spin tilt distribution is a mixture model consisting of an isotropic component and a second component in which the spins are preferentially (anti-) aligned with the orbital angular momentum (Talbot \& Thrane 2017). We note that only the BHs listed in Table 1 of Abbott et al. (2021d) are included in this population analysis.

Figure 1 shows the one-dimensional posterior probability density (PDF) of the cosine of the $\mathrm{BH}$ tilt angle for the population of $\mathrm{BBH}$ mergers (grey) alongside the tilt PDFs of the BHs in the individual NS-BH events. We take our posterior sample sets from the most recent LVK data release available (Table 1). While the $\mathrm{BH}$ spin tilts of GW191219_163120 and GW200105 are indistinguishable from the prior, the BH spin tilts of GW190426_152155, GW190917_114630 and GW200115 show posterior support that is distinct from the prior and the observed $\mathrm{BH}$ population. Since the spin measurements are dominated by the primary object and the tilt angle is expected to be largely unchanged after the first supernova (SN) (e.g. Gerosa et al. 2018), such a difference may reflect discrepancies in either the pre$\mathrm{SN}$ evolution or the magnitude of the natal kicks experienced by the two populations. In particular, $\mathrm{BHs}$ at the higher end of the $\mathrm{BBH}$ mass distribution likely come from distinct progenitor populations, and form via direct collapse with very little mass ejection and highly suppressed natal kicks.

For a quantitative measure of the statistical difference between the mean BH tilt PPD and the one-dimensional PDF for the five NSBH candidates, we employ the Jensen-Shannon divergence ( $D_{\mathrm{JS}}$; Lin 1991, see Table 1). We find a departure between the measured spin tilt posteriors of GW200115, GW190917_114630 and GW190426_152155 and the median of the observed astrophysical $\mathrm{BH}$ population from the $\mathrm{BBH}$ events. The median values of the spin tilt posteriors for these three events are $\approx-0.55$, compared to +0.24 for the BBH population. This suggests support for the hypothesis that the BHs of at least GW200115, GW190917_114630 and GW190426_152155 stand out from the observed population. The BH tilt posteriors for GW200105 and GW191219_163120, on the other hand, are uninformative relative to the spin tilt prior and fully consistent with the population PPD. The cumulative distribution functions (CDFs) of GW200115, GW190917_114630 and GW190426_152155 show 79.9, 72.6 and 74.2 per cent of the total probability in favour of tilts greater than 90 degrees $(\cos (\theta) \leq 0)$, respectively.

It is important to note here that all of the statements we make about the posterior distributions are sensitive to the choice of priors (see e.g. Vitale et al. 2017b; Mandel \& Fragos 2020; Zevin et al. 2020). These are uniform in spin magnitude and isotropic in spin orientation (Abbott et al. 2021b). Different assumptions about the prior may produce different posterior distributions, though quantifying this effect is beyond the scope of this paper. 


\begin{tabular}{cccccccc}
\hline \hline Event & $\begin{array}{c}\text { Tilt } \\
\cos (\theta)\end{array}$ & $D_{\text {JS }}$ & $\begin{array}{c}\mathrm{m}_{1} \\
\mathrm{M}_{\odot}\end{array}$ & $\begin{array}{c}\mathrm{m}_{2} \\
\mathrm{M}_{\odot}\end{array}$ & $\begin{array}{c}\mathrm{m}_{\text {tot }} \\
\mathrm{M}_{\odot}\end{array}$ & Release & Sample set \\
\hline GW190426_152155 & $-0.53_{-0.43}^{+1.36}$ & 0.17 & $5.7_{-1.5}^{+3.9}$ & $1.5_{-0.5}^{+0.8}$ & $7.2_{-1.5}^{+3.5}$ & GWTC-2 & PRECESSINGSPINIMRHM \\
GW190917_114630 & $-0.54_{-0.44}^{+1.27}$ & 0.17 & $9.3_{-4.4}^{+3.4}$ & $2.1_{-0.5}^{+1.5}$ & $11.4_{-2.9}^{+3.0}$ & GWTC-2.1 & PRECESSINGSPINIMRHM \\
GW191219_163120 & $0.00_{-0.86}^{+0.74}$ & 0.07 & $31.1_{-2.8}^{+2.2}$ & $1.17_{-0.06}^{+0.07}$ & $32.3_{-2.7}^{+2.2}$ & GWTC-3 & C01:MIXED \\
GW200105 & $0.01_{-0.93}^{+0.90}$ & 0.03 & $9.0_{-1.7}^{+1.5}$ & $1.91_{-0.24}^{+0.33}$ & $11.0_{-1.4}^{+1.5}$ & GWTC-3 & C01:MIXED \\
GW200115 & $-0.56_{-0.40}^{+1.15}$ & 0.22 & $5.9_{-2.5}^{+2.0}$ & $1.44_{-0.29}^{+0.85}$ & $7.4_{-1.7}^{+1.8}$ & GWTC-3 & C01:IMRPHENOMXPHM:HIGHSPIN \\
\hline \hline
\end{tabular}

Table 1. GW events taken to be NS-BH merger candidates in this analysis. Data presented are median values with 90 per cent credible intervals.

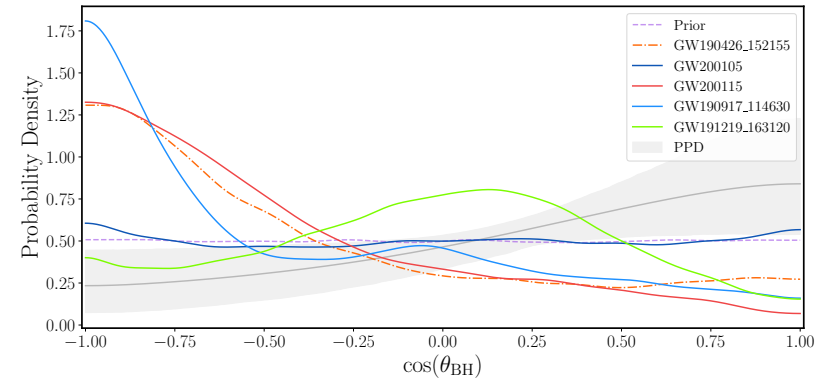

Figure 1. Posterior predictive distribution (PPD) of the $\mathrm{BH}$ tilt angles assuming the default spin model of Abbott et al. (2021d). The BH spins of GW200115 (red), GW190426_152155 (orange dashed) and GW190917_114630 (light blue) clearly stand out from the BH population (grey), where the solid grey line is the mean of the PPD and the shaded region demarcates the 5th and 95th quantile.

\section{CONSTRAINTS ON COMPACT BINARY EVOLUTION}

The dominant channel for producing compact binary mergers is expected to be isolated binary evolution, wherein the two stars are born, evolve, and merge without outside interaction. This channel predicts merger rates of $\sim$ few $\times 1-100 \mathrm{Gpc}^{-3} \mathrm{yr}^{-1}$, consistent with the current population of GW-detected NS-BH mergers (Dominik et al. 2013; Mapelli \& Giacobbo 2018; Wysocki et al. 2018; Broekgaarden et al. 2021; Broekgaarden \& Berger 2021). Rates lower by 1-2 orders of magnitude are predicted from formation in globular clusters (Clausen et al. 2013) or from isolated triples (Fragione \& Loeb 2019). However, given current model uncertainties, significant contributions to the merger rate could also arise from dynamical interactions in young stellar clusters (Rastello et al. 2020; Santoliquido et al. 2020; but see Fragione \& Banerjee 2020; Hoang et al. 2020) and the disks of active galactic nuclei (McKernan et al. 2020c).

Broadly, the 'classic' isolated binary channel proceeds as follows (e.g. Smarr \& Blandford 1976; Srinivasan 1989; Belczynski et al. 2002): the binary is born with the component spins roughly aligned with the orbit. The more massive (primary) component evolves to fill its Roche lobe, and initiates stable mass transfer onto the secondary. Eventually, the primary star ends its life as a core-collapse SN and forms the first compact object, which is usually a black hole. The SN imparts a natal kick on the primary, which can unbind over 95 per cent of wide binaries (e.g. Renzo et al. 2019). This natal kick is thought to be the dominant influence on the eventual tilt of the primary (Gerosa et al. 2018), with the tilt largely unchanged from this point onward.

If the binary survives the kick, the secondary star will later evolve to fill its Roche lobe and initiate mass transfer onto the primary. This transfer can become unstable, and result in a common envelope (CE) phase (e.g. Ivanova et al. 2013). The CE rapidly decreases the binary separation through viscous drag. If the $\mathrm{CE}$ can be ejected before a merger occurs, the subsequent core-collapse SN of the secondary results in a tight compact object binary that will eventually merge as its orbital angular momentum is lost to GW emission.

\subsection{Natal kicks}

In the absence of direct observations of $\mathrm{BH}$ velocities, the distribution of their natal kicks is often assumed to be a modified version of the NS natal kick distribution. This is typically taken to be a Maxwellian distribution with a dispersion of $\sigma=265 \mathrm{~km} / \mathrm{s}$, based on the observed velocities of pulsars in the Milky Way (Hobbs et al. 2005). Rodriguez et al. (2016) explored the BBH tilt angles induced by natal kicks with three different prescriptions for converting the observed NS kick distribution into a BH kick distribution:

(i) "Fallback kicks", wherein some fraction $f_{\mathrm{fb}}$ of the ejected mass (proportional to the core mass of the progenitor star) will "fall back" onto the $\mathrm{BH}$, damping its velocity by $V_{\mathrm{kick}}^{\mathrm{BH}}=\left(1-f_{\mathrm{fb}}\right) V_{\mathrm{kick}}^{\mathrm{NS}}$.

(ii) "Proportional kicks", where the $\mathrm{BH}$ natal kick is modified in proportion with the maximum mass of an NS by $V_{\mathrm{kick}}^{\mathrm{BH}}=\left(\frac{m_{\mathrm{NS}}}{m_{\mathrm{BH}}}\right) V_{\text {kick }}^{\mathrm{NS}}$.

(iii) "Full kicks", where $V_{\text {kick }}^{\mathrm{BH}}=V_{\text {kick }}^{\mathrm{NS}}$.

Each prescription was assessed for an isotropic distribution of kicks, and a 'polar' distribution confined to a cone with a half opening angle of $10^{\circ}$ from the star's spin axis. Polar kicks are motivated by the observed relationship between the rotation axis and direction of motion in some pulsars (e.g. Johnston et al. 2005; Yao et al. 2021). The BBH population was created by evolving a stellar population with the Binary Stellar Evolution (BSE; Hurley et al. 2002) code. This parent population featured a range of metallicities between $0.005 \mathrm{Z}_{\odot}$ and $1.5 \mathrm{Z}_{\odot}$. Primary masses were drawn from a Kroupa (2001) initial mass function ranging between $18 \mathrm{M}_{\odot}$ and $150 \mathrm{M}_{\odot}$, with a flat mass ratio distribution between 0 and 1 . The semi-major axes of the binaries were drawn from a flat distribution in log space between $10 \mathrm{R}_{\odot}$ and $10^{5} \mathrm{R}_{\odot}$. The final sample of BBHs was limited to only those that would merge via gravitational-wave emission within 13.8 Gyr. The tilt of the orbit after each natal kick is calculated using the formalism developed in the appendix of Hurley et al. (2002). After being kicked, each binary was evolved (Gerosa \& Kesden 2016) until it entered the LIGO band $(10 \mathrm{~Hz})$, at which point the tilts are assessed. For further discussion on the effects of natal kicks on binary orbits and how spin-orbit tilt can be calculated, see e.g. Tauris \& Takens (1998); Brandt \& Podsiadlowski (1995); Kalogera (1996, 2000).

Using the data of Rodriguez et al. (2016), we investigate the tilt of the primary star as a function of its mass for each kick prescription (Figure 2). Data are grouped into bins of $2 \mathrm{M}_{\odot}$, which is the cause of 

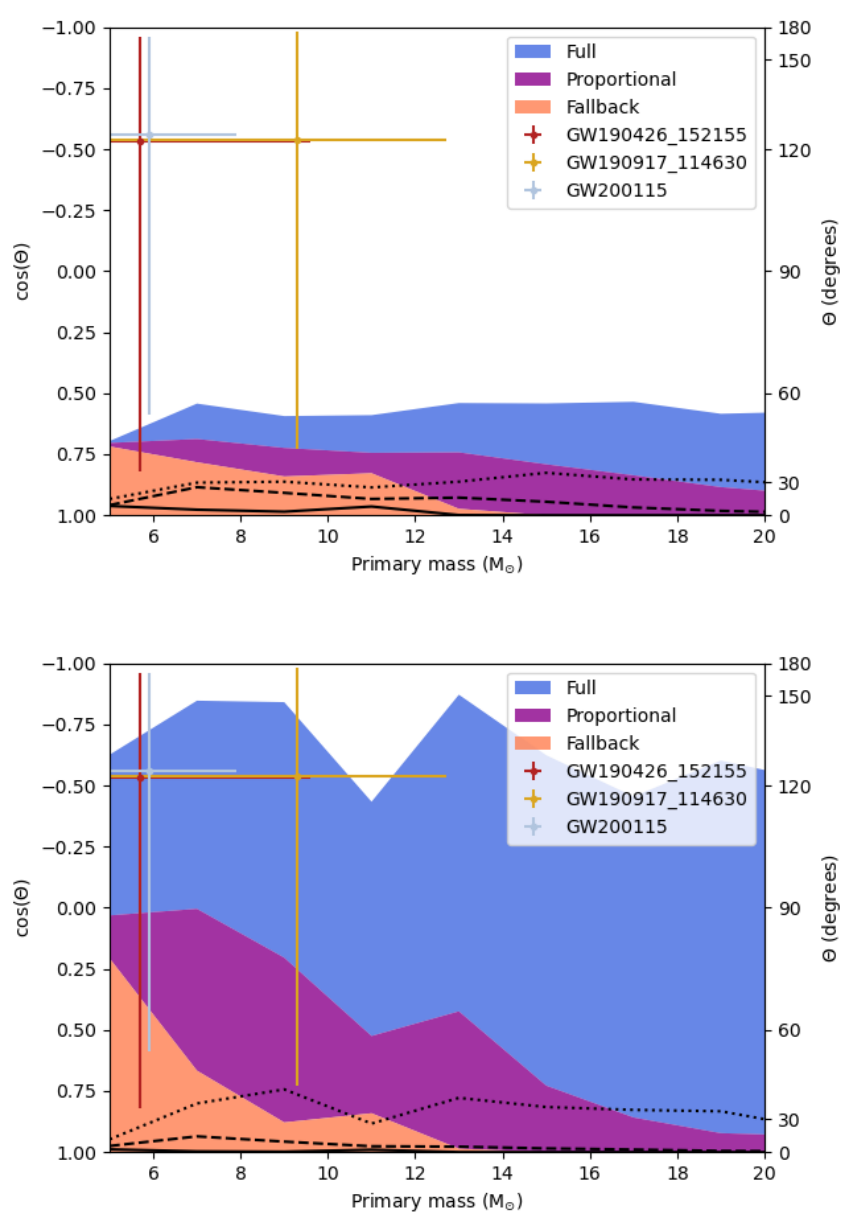

Figure 2. The spin-orbit tilt angles induced on a nascent $\mathrm{BH}$ as a result of the natal kick it receives during $\mathrm{SN}$ as a function of its mass for primary stars considered in Rodriguez et al. (2016). Shaded regions show where the lower 90 per cent of sources lie for three different kick prescriptions, with the median tilts for each indicated by the solid, dashed and dotted lines for the fallback, proportional and full kicks, respectively. Data are grouped into bins of $2 \mathrm{M}_{\odot}$.

The upper panel is for polar kicks, and the lower for an isotropic distribution. The 90 per cent confidence intervals for the primary mass and tilt is shown for GW200115, GW190917_114630 and GW190426_152155.

the 'spiky' curve. Polar kicks produce misalignments of $\cos (\theta)>0.5$ in almost all cases, including for full kicks. While only the "isotropic full kicks" prescription produces significant tilt across the full range of masses, the "isotropic fallback" and "isotropic proportional" kick prescriptions can produce significant, even anti-aligned, tilts for lower mass stars. If tidal forces can efficiently realign the spins with the orbital plane, the expected tilt angles are greatly reduced (Rodriguez et al. 2016). However, tidal processes will preferentially work to align the spin of the less massive object, which is usually the second SN (Gerosa et al. 2018), whereas here we are interested in the more massive object (since it is the tilt of the $\mathrm{BH}$ that is well measured).

We consider the three events that show significant posterior divergence from both the prior and the $\mathrm{BBH}$ tilt distribution (Table 1). The 90 per cent confidence interval of the tilt of GW200115 is inconsistent with the 90th centile of tilts for an isolated binary evolution channel that favours polar natal kicks, regardless of which prescrip- tion is used.GW190917_114630 is inconsistent with the proportional and fallback tilt distributions. The 90 per cent confidence interval of the tilt of GW190426_152155 does marginally overlap with the 90th centile of tilts for the polar kick distributions. Considering the three events together, we conclude that a polar kick distribution for BHs is disfavoured by the $\mathrm{GW}$ observations. Although we are comparing NS-BH mergers to BBH mergers here, Figure 2 demonstrates that tilt can be achieved via natal kicks in lower mass BHs, while in higher mass cases these natal kicks are suppressed either by fallback material or conservation of momentum arguments. We expect $\mathrm{BBH}$ primaries with masses comparable to the BHs in our NS-BH events to be directly comparable. This may not be the case for higher mass systems, which could arise through different evolutionary pathways.

When compared to the isotropic kick distributions (Figure 2, lower), the peak probabilities of the posterior tilt distributions of GW200115, GW190917_114630 and GW190426_152155 are towards the edges of the $90^{\text {th }}$ centile of tilts produced by $\mathrm{BH}$ natal kicks, although their confidence intervals extend to more moderate tilts. GW190917_114630 is inconsistent with the fallback distribution. Having three of the first five known events (with the other two unconstrained) towards the edge of the expected tilt distribution is not impossible, but it may hint at either another mechanism by which tilt can be induced, or point towards higher-than-expected BH natal kick velocities. The latter conclusion was also suggested to explain the tilt of the low-mass BBH GW151226 (O'Shaughnessy et al. 2017), reconcile the relative rates of $\mathrm{BBH}$ and binary NS mergers detected in GW (Chruslinska et al. 2018), align the predicted and observed spin distributions of the BBH population (Callister et al. 2020) and in independent analyses of GW200105 and GW200115 (Fragione et al. 2021). Some estimates of BH velocities from low mass X-ray binaries imply velocities of $80 \mathrm{~km} \mathrm{~s}^{-1}$ or less (Mandel 2016), though other studies prefer higher kick velocities of $>100 \mathrm{~km} \mathrm{~s}^{-1}$ (Repetto et al. 2017). Alternatively, Zhu (2021) suggest that large tilt angles could be achieved in GW200115 with an extremely high velocity $(600 \mathrm{~km} / \mathrm{s})$ natal kick to the NS during the second SN in the binary if it were directed within $30^{\circ}$ of the pre-SN orbital plane.

\subsection{Additional tilt from stable mass transfer}

Stegmann \& Antonini (2021) explore the effects of stable mass transfer prior to the first SN on the tilt angle of BBH systems. Mass transfer is shown to slowly tilt the donor (primary) star from an (assumed) initially aligned configuration $(\cos (\theta)=0.9-1.0)$ until the spin is significantly misaligned $(\cos (\theta) \sim 0)$. End-to-end simulations accounting for mass transfer, natal kicks, tidal forces, and the CE phase result in a population of $\mathrm{BBH}$ mergers with a tilt angle distribution that peaks at an aligned configuration, but remains relatively flat between $\cos (\theta)=1$ and $\cos (\theta)=0$. If tides are weak, this distribution may even become bimodal around $\cos (\theta)=1$ and $\cos (\theta)=0$. Antialigned spins $(\cos (\theta)<0)$ remain comparatively rare; an order of magnitude or more less common.

The median tilts of our sample with significant divergence between posterior and prior (Table 1) lie well away from the peaks of the tilt angle probability distributions discussed in Stegmann \& Antonini (2021). However, the 90 per cent confidence intervals are broad, and consistent with $\cos (\theta)=0$, where the probability rapidly rises. The inclusion of tilt via stable mass transfer therefore serves to ease the tension between the expectations for BH kicks and the peak posterior measurements. However, the peaks of the distributions still favour tilts that are greater than would be expected from a combination of canonical BH kick velocities and tilting via stable mass transfer cf. Stegmann \& Antonini (2021). 


\subsection{Observational implications for binary evolution physics}

Independent of its potential to induce tilt, stable mass transfer prior to the first SN has important effects on the mass distribution and merger rate of NS-BH binaries (Kruckow et al. 2018; Broekgaarden et al. 2021; Shao \& Li 2021). In particular, when accretion of the mass lost by the primary onto the secondary is efficient, binaries following the 'classic' channel (see Section 3) tend to merge before they can become compact binaries (Broekgaarden et al. 2021). This leads to a reduction of the overall NS-BH merger rate, and a rise in the relative rates of non-traditional channels such as those that avoid $\mathrm{CE}$ (or alternatively experience it twice), or those that proceed via chemically homogeneous evolution (e.g. Marchant et al. 2017).

We emphasize that the predictions for a higher efficiency stable mass transfer pathway are in good agreement with the observations of the first two confirmed NS-BH mergers, and the candidates GW190426_151255 and GW190917_114630. In particular, Model $\mathrm{C}$ in Broekgaarden et al. (2021), which fixes the fraction of mass lost by the donor that is accreted by the secondary to $\beta=0.5$, predicts that the GW-detected distribution of NS-BH mergers will peak at a total binary mass of $\approx 7 \mathrm{M}_{\odot}$, with a preference for mass ratios of $q \approx 3$. This compares favourably to our sample (Table 1 ), particularly GW190426_151255 and GW200115. The exception is GW191219_163120, which has a $31.1 \mathrm{M}_{\odot} \mathrm{BH}$ that is not well explained by any of the progenitor pathway models. Furthermore, the event-based NS-BH merger rate derived in Abbott et al. (2021e) from GW200105 and GW200115 is fully consistent with the expected merger rate of Model C in Broekgaarden et al. (2021), although both exhibit broad distributions.

The early NS-BH merger observations may therefore suggest that the 'classic $\mathrm{CE}$ ' formation channel where binaries undergo one $\mathrm{CE}$ phase may be less common than expected in NS-BH events. If orbital decay is indeed being facilitated in many cases by efficient stable mass transfer or two common envelope phases, then future NS-BH merger events (e.g. during the LVK O4 run) will continue to show total binary masses distributed around $7 \mathrm{M}_{\odot}$. This is in contrast to the mass distribution in the fiducial model of Broekgaarden et al. (2021), which peaks at around $15 \mathrm{M}_{\odot}$ (in part because more massive systems should be easier to detect in GW). A preference for lower mass $\left(5-15 \mathrm{M}_{\odot}\right) \mathrm{BHs}$ (and higher mass NSs), compatible with the observed NS-BH sample, was also shown in Giacobbo \& Mapelli (2018).

Conversely, some dynamical capture channels suggest NS-BH mergers that are significantly more massive than expected for the isolated binaries (e.g. Rastello et al. 2020). This pathway may provide a natural explanation for GW191219_163120. Rastello et al. (2020) find that roughly 25 per cent of NS-BH mergers resulting from dynamical capture will have a total binary mass of $>30 \mathrm{M}_{\odot}$. They estimate the overall rate of mergers formed in this way to be $\sim 28 \mathrm{Gpc}^{-3} \mathrm{yr}^{-1}$, a factor of $\sim 2$ less than the isolated evolution merger rate of $\sim 49 \mathrm{Gpc}^{-3} \mathrm{yr}^{-1}$ (Santoliquido et al. 2020). Therefore, our relative observed rates of four NS-BH mergers consistent with isolated binary evolution to one very massive $\left(m_{\text {tot }}>30 \mathrm{M}_{\odot}\right)$ NS-BH merger potentially driven by dynamical capture is in good agreement with the rates derived by Rastello et al. (2020) and Santoliquido et al. (2020).

Predictions from population synthesis modelling for the spin magnitude of the $\mathrm{BH}$ at the point of merger are uncertain. A common prediction for $\mathrm{BBH}$ evolution is that a $\mathrm{BH}$ will have essentially zero spin at the point of merger, having lost most of its angular momentum to stellar winds, mass transfer or angular momentum transport at the point of collapse (e.g. Qin et al. 2018; Fuller \& Ma 2019). It should be noted that despite supporting anti-aligned tilts, the broad posterior spin distributions of GW190426_152155, GW190917_114630 and GW200115 are consistent with this picture. However, highlyspinning and anti-aligned BBHs are expected in cases with weak stellar core-envelope coupling or through tidal synchronisation (Steinle \& Kesden 2021). A BH may also be spun up prior to its collapse if it is born second, resulting in a large spin at the point of merger (Bavera et al. 2020; Chattopadhyay et al. 2021). Ultimately, high mass X-ray binaries (Miller \& Miller 2015) and long GRBs (e.g. Fryer et al. 2019) both provide evidence that some BHs must be rapidly rotating in binary systems at some point in their evolution. The true picture is awaiting observational confirmation, although given the degeneracies in the GW posteriors, associated EM constraints will likely be required to uniquely identify the characteristics of the pre-merger binary.

\section{IMPLICATIONS FOR MULTI-MESSENGER EVENTS}

NS-BH mergers are a potential source of EM emission if the NS is disrupted during inspiral, such that some of the material remains outside of the BH event horizon. Hot, dense, and neutron-rich material could facilitate rapid neutron capture (r-process; Lattimer \& Schramm 1974; Eichler et al. 1989; Freiburghaus et al. 1999) nucleosynthesis, which would lead to a thermal transient known as a kilonova (KN; Li \& Paczyński 1998; Rosswog 2005; Metzger et al. 2010; Barnes \& Kasen 2013; Metzger 2017) when the newly-formed unstable heavy elements flow away from the merger site in winds and dynamical ejecta, and decay to stability. If a few tenths of a solar mass accrete onto the $\mathrm{BH}$ then the merger may also power a short GRB, if a relativistic jet can be launched successfully (Blandford \& Znajek 1977; Barbieri et al. 2019; Gompertz et al. 2020b).

The dominant properties that predict the remnant mass outside of the $\mathrm{BH}$ event horizon are the orbit-aligned component of the spin of the pre-merger $\mathrm{BH}\left(\chi_{\mathrm{BH}}\right)$ and the mass ratio of the binary, $q$. The compactness of the NS is also important. Figure 3 shows the expected remnant mass (cf. Foucart et al. 2018) as a function of $\chi_{\mathrm{BH}}$ and $q$, assuming an NS with $M=1.4 \mathrm{M}_{\odot}$ and a radius of $12 \mathrm{~km}$. Essentially no mass is expected to remain outside of the event horizon for any of our NS-BH candidates, making the prospect of an EM accompaniment unlikely. We note that follow-up of these four events during $\mathrm{O} 3$ did not yield any detected counterparts (Hosseinzadeh et al. 2019; Lundquist et al. 2019; Gompertz et al. 2020a; Paterson et al. 2020; Anand et al. 2021). While this outcome is in line with the expected absence of an EM transient from modelling (see also Zhu et al. 2021), efforts to constrain one were also hindered by a combination of poor localisations (1300, 2100, 7700, 900 square degrees, 90 per cent containment) and large distances $\left(370_{-300}^{+320}, 720_{-310}^{+340}, 280_{-110}^{+110}, 300_{-100}^{+150} \mathrm{Mpc}\right)$ for GW190426_152155, GW190917_114630, GW200105 and GW200115 respectively (Abbott et al. 2021e,b; The LIGO Scientific Collaboration et al. 2021a).

If NS-BH mergers do show low $\chi_{\mathrm{BH}}$, whether due to a low spin magnitude or large tilt, the prospects for NS-BH-driven $\mathrm{KNe}$ and GRBs are poor. However, Figure 3 demonstrates that with a modest increase in the GW-measured spin, EM observations could significantly tighten the constraints on $\chi_{\mathrm{BH}}$ and $q$ by independently detecting or ruling out a transient. This possibility has important implications for constraining the properties of nuclear matter through multi-messenger observations even where no detection is made. Furthermore, the presence or absence of EM-bright NS-BH mergers provides an immediate constraint on their average spins, and therefore their progenitor pathways, as discussed in Section 3.3. 


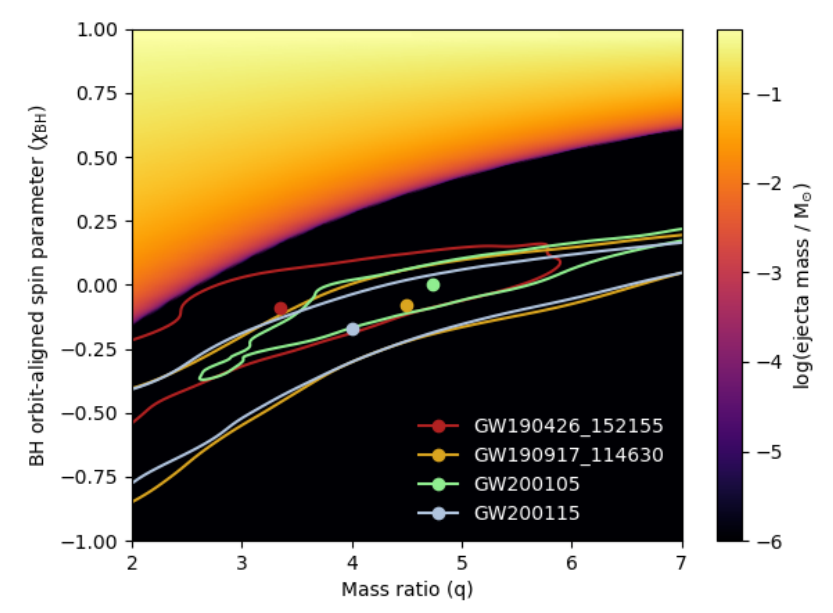

Figure 3. The post-merger remnant mass as a function of the orbit-aligned component of the $\mathrm{BH}$ spin $\left(\chi_{\mathrm{BH}}\right)$ and the binary mass ratio $(q)$ (cf. Foucart et al. 2018). An NS with $m_{2}=1.4 \mathrm{M}_{\odot}$ and $r=12 \mathrm{~km}$ is assumed. Contours show the 90 per cent credible intervals for the NS-BH events and candidates.

\section{CONCLUSIONS}

We investigate the tilt angles of the recently announced NS-BH merger events GW200105 and GW200115 (Abbott et al. 2021e) and the candidates GW190426_152155, GW190917_114630 and GW191219_163120, and compare them to the tilt angles derived for the known population of BBH events (Abbott et al. 2021d). The posterior tilt distributions of GW200115, GW190917_114630 and GW190426_152155 place them in a low-probability region of the posterior predictive distribution of $\mathrm{BBH}$ tilt angles, with peaks that favour anti-aligned spins (though the distributions are broad). The tilt angles of GW200105 and GW191219_163120 are unconstrained. Since the measurements are dominated by the properties of the primary in all binaries, the tilts measured at the point of merger are largely set following the first SN (assuming isolated binary evolution and that the $\mathrm{BH}$ formed first). Hence, the discrepancy in the populations may reflect differences in the pre-SN evolution or the distribution of natal kick velocities.

The peaks of the posterior tilt distributions in these events are in tension with canonical assumptions for $\mathrm{BH}$ natal kicks where velocities are either attenuated by post-SN fallback of material or modified in proportion with the BH mass (cf. Rodriguez et al. 2016). If real, this may be resolved via BHs that are imbued with kick velocities of comparable magnitudes to NSs (see also O'Shaughnessy et al. 2017; Repetto et al. 2017; Chruslinska et al. 2018; Atri et al. 2019; Fragione et al. 2021), extreme NS kick velocities (Zhu 2021), and/or extra tilt induced by stable mass transfer (Stegmann \& Antonini 2021). However, the broad distributions also extend to more moderate tilts within their 90 per cent confidence intervals. We also show that the distribution of kicks are likely isotropic, since preferentially polar kicks (motivated by the observed correlation between the kick direction and spin axis of newly formed pulsars; Johnston et al. 2005; Yao et al. 2021) are inefficient at producing tilt.

Independent of our considerations for the tilt angles, the early sample of NS-BH events and candidates display lower total binary masses and mass ratios than expected under typical assumptions for stellar evolution. These properties are consistent with an evolutionary pathway that features efficient stable mass transfer prior to the first
SN, e.g. Model C in Broekgaarden et al. (2021). The rates predicted by this model also match the event-based rate of NS-BH mergers in Abbott et al. (2021e). If this is a general property of binary evolution then the 'classic' channel may be much less common than is typically assumed, with more massive stellar binaries merging before they become compact objects. We predict that future NS-BH detections in GW will continue to be distributed around a total binary mass of $\sim 7 \mathrm{M}_{\odot}$ and a mass ratio of $q \approx 3$ if this is the correct interpretation.

If the suggestion of highly tilted BHs in NS-BH mergers is real then the prospects for EM accompaniments are poor, since the amount of material that is stripped from the NS before merger is a function of the orbit-aligned spin component of the BH. Anti-aligned spins like the ones found at the peaks of the posterior distributions for GW200115, GW190917_114630 and GW190426_152155 are particularly detrimental to the prospects for multi-messenger observations of NS-BH binaries (e.g. Foucart et al. 2018). However, the weak constraints on key EM-predictive properties like $\chi_{\mathrm{BH}}$ and $q$ demonstrate the need for future studies that link GW signals to their expected EM counterparts. In tandem, these can provide much stricter constraints on the parameter space, helping to break degeneracies in the GW measurements.

We suggest that the evidence from the early LVK observations of NS-BH spins, masses and rates points towards a high mass transfer efficiency and strong isotropic BH natal kicks, which are both key ingredients for future population synthesis models.

\section{ACKNOWLEDGEMENTS}

We thank the anonymous referee for careful consideration of this manuscript and for suggestions that improved the detail and scope of our study. We thank Ilya Mandel, Davide Gerosa, Floor Broekgaarden and Edo Berger for useful discussions on binary evolution physics, Chris Moore for help with the statistical interpretation, and Carl Rodriguez for providing simulation data. $\mathrm{MN}$ is supported by a Royal Astronomical Society Research Fellowship. MN and BG acknowledge funding by the European Research Council (ERC) under the European Union's Horizon 2020 research and innovation programme (grant agreement No. 948381). AV acknowledges the support of the Royal Society and Wolfson Foundation. This research has made use of data, software and/or web tools obtained from the Gravitational Wave Open Science Center (https://www.gw-openscience.org/ ), a service of LIGO Laboratory, the LIGO Scientific Collaboration and the Virgo Collaboration. LIGO Laboratory and Advanced LIGO are funded by the United States National Science Foundation (NSF) as well as the Science and Technology Facilities Council (STFC) of the United Kingdom, the Max-Planck-Society (MPS), and the State of Niedersachsen/Germany for support of the construction of Advanced LIGO and construction and operation of the GEO600 detector. Additional support for Advanced LIGO was provided by the Australian Research Council. Virgo is funded, through the European Gravitational Observatory (EGO), by the French Centre National de Recherche Scientifique (CNRS), the Italian Istituto Nazionale di Fisica Nucleare (INFN) and the Dutch Nikhef, with contributions by institutions from Belgium, Germany, Greece, Hungary, Ireland, Japan, Monaco, Poland, Portugal, Spain.

\section{DATA AVAILABILITY}

The data used in this publication are available from the authors upon reasonable request. 
The posterior samples for GW190426_152155 are from GWTC-2 (Abbott et al. 2021b) and can be accessed at https://dcc.ligo.org/LIGO-P2000223/public/ under sample set PRECESSINGSPINIMRHM.

The posterior samples for GW190917_114630 are from GWTC2.1 (The LIGO Scientific Collaboration et al. 2021a) and can be accessed at https://zenodo.org/record/5117703\#.YZYe6FOnzuR under sample set PRECESSINGSPINIMRHM.

The posterior samples for GW191219_163120, GW200105 and GW200115 are from GWTC-3 (The LIGO Scientific Collaboration et al. 2021b) and can be accessed at https://zenodo.org/record/5546663\#.YZYO41Ony8U under sample set C01:MIXED for GW191219_163120 and GW200105, and sample set C01:IMRPhENOMXOHM:HighSPIN for GW200115 (for which no mixed samples were available).

\section{REFERENCES}

Abbott B. P., et al., 2016, Phys. Rev. Lett., 116, 061102 Abbott B. P., et al., 2017a, Phys. Rev. Lett., 119, 161101 Abbott B. P., et al., 2017b, ApJ, 848, L12

Abbott B. P., et al., 2017c, ApJ, 848, L13

Abbott R., et al., 2020a, Phys. Rev. Lett., 125, 101102

Abbott R., et al., 2020b, ApJ, 896, L44

Abbott R., et al., 2021a, https://dcc.ligo.org/LIGO-P2000434/ public

Abbott R., et al., 2021b, Physical Review X, 11, 021053

Abbott R., et al., 2021c, SoftwareX, 13, 100658

Abbott R., et al., 2021d, ApJ, 913, L7

Abbott R., et al., 2021e, ApJ, 915, L5

Acernese F., et al., 2015, Classical and Quantum Gravity, 32, 024001

Anand S., et al., 2021, Nature Astronomy, 5, 46

Andreoni I., et al., 2017, Publ. Astron. Soc. Australia, 34, e069

Antoniadis J., et al., 2013, Science, 340, 448

Arca Sedda M., 2020, Communications Physics, 3, 43

Arcavi I., et al., 2017, ApJ, 848, L33

Atri P., et al., 2019, MNRAS, 489, 3116

Barbieri C., Salafia O. S., Perego A., Colpi M., Ghirlanda G., 2019, A\&A, 625, A152

Barnes J., Kasen D., 2013, ApJ, 775, 18

Bavera S. S., et al., 2020, A\&A, 635, A97

Belczynski K., Kalogera V., Bulik T., 2002, ApJ, 572, 407

Blandford R. D., Znajek R. L., 1977, MNRAS, 179, 433

Brandt N., Podsiadlowski P., 1995, MNRAS, 274, 461

Breschi M., Perego A., Bernuzzi S., Del Pozzo W., Nedora V., Radice D., Vescovi D., 2021, arXiv e-prints, p. arXiv:2101.01201

Broekgaarden F. S., Berger E., 2021, arXiv e-prints, p. arXiv:2108.05763

Broekgaarden F. S., et al., 2021, arXiv e-prints, p. arXiv:2103.02608

Callister T. A., Farr W. M., Renzo M., 2020, arXiv e-prints, p. arXiv:2011.09570

Chattopadhyay D., Stevenson S., Hurley J. R., Bailes M., Broekgaarden F., 2021, MNRAS, 504, 3682

Chornock R., et al., 2017, ApJ, 848, L19

Chruslinska M., Belczynski K., Klencki J., Benacquista M., 2018, MNRAS, 474, 2937

Clausen D., Sigurdsson S., Chernoff D. F., 2013, MNRAS, 428, 3618

Coughlin M. W., Dietrich T., Margalit B., Metzger B. D., 2019, MNRAS, 489, L91

Coulter D. A., et al., 2017, Science, 358, 1556

Cowperthwaite P. S., et al., 2017, ApJ, 848, L17

Cromartie H. T., et al., 2020, Nature Astronomy, 4, 72

D’Avanzo P., et al., 2018, A\&A, 613, L1

Demorest P. B., Pennucci T., Ransom S. M., Roberts M. S. E., Hessels J. W. T., 2010, Nature, 467, 1081
Dietrich T., Coughlin M. W., Pang P. T. H., Bulla M., Heinzel J., Issa L., Tews I., Antier S., 2020, arXiv e-prints, p. arXiv:2002.11355

Dominik M., Belczynski K., Fryer C., Holz D. E., Berti E., Bulik T., Mandel I., O'Shaughnessy R., 2013, ApJ, 779, 72

Drout M. R., et al., 2017, Science, 358, 1570

Eichler D., Livio M., Piran T., Schramm D. N., 1989, Nature, 340, 126

Evans P. A., et al., 2017, Science, 358, 1565

Farr W. M., Sravan N., Cantrell A., Kreidberg L., Bailyn C. D., Mandel I., Kalogera V., 2011, ApJ, 741, 103

Farr W. M., Stevenson S., Miller M. C., Mandel I., Farr B., Vecchio A., 2017, Nature, 548, 426

Fernández R., Foucart F., Lippuner J., 2020, MNRAS, 497, 3221

Foucart F., Hinderer T., Nissanke S., 2018, Phys. Rev. D, 98, 081501

Fragione G., Banerjee S., 2020, ApJ, 901, L16

Fragione G., Loeb A., 2019, MNRAS, 486, 4443

Fragione G., Loeb A., Rasio F. A., 2021, arXiv e-prints, p. arXiv:2108.06538 Freiburghaus C., Rosswog S., Thielemann F.-K., 1999, ApJ, 525, L121

Fryer C. L., Lloyd-Ronning N., Wollaeger R., Wiggins B., Miller J., Dolence J., Ryan B., Fields C. E., 2019, European Physical Journal A, 55, 132

Fuller J., Ma L., 2019, ApJ, 881, L1

Gerosa D., Kesden M., 2016, Phys. Rev. D, 93, 124066

Gerosa D., Berti E., O'Shaughnessy R., Belczynski K., Kesden M., Wysocki D., Gladysz W., 2018, Phys. Rev. D, 98, 084036

Giacobbo N., Mapelli M., 2018, MNRAS, 480, 2011

Goldstein A., et al., 2017, ApJ, 848, L14

Gompertz B. P., et al., 2020a, MNRAS, 497, 726

Gompertz B. P., Levan A. J., Tanvir N. R., 2020b, ApJ, 895, 58

Hallinan G., et al., 2017, Science, 358, 1579

Hoang B.-M., Naoz S., Kremer K., 2020, ApJ, 903, 8

Hobbs G., Lorimer D. R., Lyne A. G., Kramer M., 2005, MNRAS, 360, 974 Hosseinzadeh G., et al., 2019, ApJ, 880, L4

Hurley J. R., Tout C. A., Pols O. R., 2002, MNRAS, 329, 897

Ivanova N., et al., 2013, A\&ARv, 21, 59

Johnston S., Hobbs G., Vigeland S., Kramer M., Weisberg J. M., Lyne A. G., 2005, MNRAS, 364, 1397

Kagra Collaboration et al., 2019, Nature Astronomy, 3, 35

Kalogera V., 1996, ApJ, 471, 352

Kalogera V., 2000, ApJ, 541, 319

Kasliwal M. M., et al., 2017, Science, 358, 1559

Kawaguchi K., Kyutoku K., Shibata M., Tanaka M., 2016, ApJ, 825, 52

Kroupa P., 2001, MNRAS, 322, 231

Kruckow M. U., Tauris T. M., Langer N., Kramer M., Izzard R. G., 2018, MNRAS, 481, 1908

Krüger C. J., Foucart F., 2020, Phys. Rev. D, 101, 103002

LIGO Scientific Collaboration et al., 2015, Classical and Quantum Gravity, 32, 074001

Lamb G. P., et al., 2019, ApJ, 870, L15

Lattimer J. M., Schramm D. N., 1974, ApJ, 192, L145

Li L.-X., Paczyński B., 1998, ApJ, 507, L59

Lin J., 1991, IEEE Transactions on Information theory, 37, 145

Lipunov V. M., et al., 2017, ApJ, 850, L1

Lundquist M. J., et al., 2019, ApJ, 881, L26

Lyman J. D., et al., 2018, Nature Astronomy, 2, 751

Mandel I., 2016, MNRAS, 456, 578

Mandel I., Fragos T., 2020, ApJ, 895, L28

Mapelli M., Giacobbo N., 2018, MNRAS, 479, 4391

Marchant P., Langer N., Podsiadlowski P., Tauris T. M., de Mink S., Mandel I., Moriya T. J., 2017, A\&A, 604, A55

Margalit B., Metzger B. D., 2017, ApJ, 850, L19

Margalit B., Metzger B. D., 2019, ApJ, 880, L15

Margutti R., et al., 2017, ApJ, 848, L20

Margutti R., et al., 2018, ApJ, 856, L18

McCully C., et al., 2017, ApJ, 848, L32

McKernan B., Ford K. E. S., O'Shaugnessy R., Wysocki D., 2020a, MNRAS, 494, 1203

McKernan B., Ford K. E. S., O'Shaughnessy R., 2020b, MNRAS, 498, 4088 McKernan B., Ford K. E. S., O'Shaughnessy R., 2020c, MNRAS, 498, 4088 Metzger B. D., 2017, Living Reviews in Relativity, 20, 3 
Metzger B. D., Arcones A., Quataert E., Martínez-Pinedo G., 2010, MNRAS, 402, 2771

Miller M. C., Miller J. M., 2015, Phys. Rep., 548, 1

Mooley K. P., et al., 2018, Nature, 554, 207

Nicholl M., et al., 2017, ApJ, 848, L18

Nicholl M., Margalit B., Schmidt P., Smith G. P., Ridley E. J., Nuttall J., 2021, MNRAS, 505, 3016

O’Shaughnessy R., Gerosa D., Wysocki D., 2017, Phys. Rev. Lett., 119, 011101

Özel F., Psaltis D., Narayan R., McClintock J. E., 2010, ApJ, 725, 1918

Paterson K., et al., 2020, arXiv e-prints, p. arXiv:2012.11700

Pian E., et al., 2017, Nature, 551, 67

Qin Y., Fragos T., Meynet G., Andrews J., Sørensen M., Song H. F., 2018, A\&A, 616, A28

Rastello S., Mapelli M., Di Carlo U. N., Giacobbo N., Santoliquido F., Spera M., Ballone A., Iorio G., 2020, MNRAS, 497, 1563

Renzo M., et al., 2019, A\&A, 624, A66

Repetto S., Igoshev A. P., Nelemans G., 2017, MNRAS, 467, 298

Rodriguez C. L., Zevin M., Pankow C., Kalogera V., Rasio F. A., 2016, ApJ, 832, L2

Rosswog S., 2005, ApJ, 634, 1202

Roulet J., Chia H. S., Olsen S., Dai L., Venumadhav T., Zackay B., Zaldarriaga M., 2021, arXiv e-prints, p. arXiv:2105.10580

Santoliquido F., Mapelli M., Bouffanais Y., Giacobbo N., Di Carlo U. N., Rastello S., Artale M. C., Ballone A., 2020, ApJ, 898, 152

Savchenko V., et al., 2017, ApJ, 848, L15

Shao Y., Li X.-D., 2021, arXiv e-prints, p. arXiv:2107.03565

Shappee B. J., et al., 2017, Science, 358, 1574

Smarr L. L., Blandford R., 1976, ApJ, 207, 574

Smartt S. J., et al., 2017, Nature, 551, 75

Soares-Santos M., et al., 2017, ApJ, 848, L16

Srinivasan G., 1989, A\&ARv, 1, 209

Stegmann J., Antonini F., 2021, Phys. Rev. D, 103, 063007

Steinle N., Kesden M., 2021, Phys. Rev. D, 103, 063032

Stevenson S., Berry C. P. L., Mandel I., 2017, MNRAS, 471, 2801

Talbot C., Thrane E., 2017, Phys. Rev. D, 96, 023012

Tanvir N. R., et al., 2017, ApJ, 848, L27

Tauris T. M., Takens R. J., 1998, A\&A, 330, 1047

The LIGO Scientific Collaboration et al., 2021a, arXiv e-prints, p. arXiv:2108.01045

The LIGO Scientific Collaboration et al., 2021b, arXiv e-prints, p. arXiv:2111.03606

Troja E., et al., 2017, Nature, 551, 71

Troja E., et al., 2018, MNRAS, 478, L18

Utsumi Y., et al., 2017, PASJ, 69, 101

Valenti S., et al., 2017, ApJ, 848, L24

Villar V. A., et al., 2017, ApJ, 851, L21

Vitale S., Lynch R., Sturani R., Graff P., 2017a, Classical and Quantum Gravity, 34, 03LT01

Vitale S., Gerosa D., Haster C.-J., Chatziioannou K., Zimmerman A., 2017b, Phys. Rev. Lett., 119, 251103

Wysocki D., Gerosa D., O’Shaughnessy R., Belczynski K., Gladysz W., Berti E., Kesden M., Holz D. E., 2018, Phys. Rev. D, 97, 043014

Yao J., et al., 2021, Nature Astronomy, 5, 788

Ye C. S., Fong W.-f., Kremer K., Rodriguez C. L., Chatterjee S., Fragione G., Rasio F. A., 2020, ApJ, 888, L10

Zevin M., Berry C. P. L., Coughlin S., Chatziioannou K., Vitale S., 2020, ApJ, 899, L17

Zhu X.-J., 2021, arXiv e-prints, p. arXiv:2108.13540

Zhu J.-P., Wu S., Yang Y.-P., Zhang B., Yu Y.-W., Gao H., Cao Z., Liu L.-D., 2021, arXiv e-prints, p. arXiv:2106.15781

This paper has been typeset from a $\mathrm{T}_{\mathrm{E}} \mathrm{X} / \mathrm{L} \mathrm{T} \mathrm{E} \mathrm{X}$ file prepared by the author. 